\title{
TELLING ENGAGING INTERACTIVE STORIES WITH EXTENDED REALITY (XR): BACK TO 1930S IN ZURICH'S MAIN TRAIN STATION
}

\author{
Yannick Pulver ${ }^{1}$, Cédric Merz ${ }^{2}$, Kathrin Koebel ${ }^{2}$, Joe Scheidegger ${ }^{3}$, Arzu Çöltekin $^{2 *}$ \\ ${ }^{1}$ Apps with love AG, Bern, Switzerland - yannick.pulver@gmail.com \\ ${ }^{2}$ University of Applied Sciences and Arts Northwestern Switzerland FHNW, Institute for Interactive Technologies IIT, \\ Brugg-Windisch, Switzerland - (cedric.merz, arzu.coltekin, kathrin.koebel)@fhnw.ch \\ ${ }^{3}$ SBB Center of Competence Extended Reality, Bern, Switzerland - joe.scheidegger@sbb.ch
}

\section{Commission IV, WG IV/9}

KEY WORDS: Mixed, Augmented, Extended Reality, location based AR, Spatial UI, User Experience, Gamification, Storytelling

\begin{abstract}
:
With increasing technical feasibility of extended reality (XR) on smartphones and tablets, we also witness increasing versatility in the use of the mixed reality (MR) (i.e., rather than augmented or virtual) in utility-oriented apps (e.g., for navigation, indoor/outdoor spatial planning, entertainment and location based gaming. In cases where user adaptation and adherence is important, the design of the story itself, visualization and interaction in the game must be engaging, and ideally support spatial knowledge acquisition. In this paper, we briefly review the literature on creating engaging MR experiences for a location based game, and present a case study in which we feature a location based MR game (SBB Stories). We conceptualized, designed and implemented the SBB Stories with user-centered design methods in collaboration with the SBB (Swiss Federal Railways). In this paper we feature an interactive story that took place in Zurich's main train station in 1937. Our findings from several cycles of user studies shows an increased spatial awareness of the surroundings as participants used the SBB Stories app. Importantly, participants reported the blending of historical and current visual elements as an outstanding and inspiring experience. Besides a high score of 83.75/100 in a standardized usability test, and a similarly high score of 4.25/5.00 in a standardized user engagement scale, all participants reported that they would spend extra time at the train station to play this game, suggesting that the app was indeed engaging.
\end{abstract}

\section{INTRODUCTION AND BACKGROUND}

As part of the 'digitalswitzerland challenge' in 2017, the Swiss Federal Railways (SBB) initiated a challenge stating that Zurich main station will become world's most digital station within two years (Leimgruber, SBB, 2017). Objectives were to offer some entertainment for those who spend time in the station, improve spatial knowledge acquisition about the station, attract people to the station and nudge them to discover the services and cultural artifacts in this space. The concept (and following development, implementation, testing) presented in this paper, i.e., the location based extended reality mobile game that blends past and present, is inspired by this initiative. In this context, the overarching goal of the research was to identify what makes an MR game engaging while supporting spatial learning, and consequently implement and test a creative concept that may be of interest to a large number of users. Note that in this paper we use the term MR when scene contains spatially registered virtual objects, AR when objects are not spatially registered (menu items etc.), and XR when for the whole system that includes both AR and MR elements. Thus, in this paper, after a brief review of location based MR games and the gamification approaches that might engage users; we present the resulting interactive MR game $S B B$ Stories, in which the player can experience stories, solve quests and travel to unexpected places and times on an adventure through Zurich main station. The overarching objective was to identify elements of design and interaction that engages people in smartphone MR games while increasing spatial awareness of their surroundings; whereas a specific question was how to attract and maintain the

\footnotetext{
* Corresponding author: arzu.coltekin@ fhnw.ch
}

attention of commuters at Zurich's main station: A usable system may not be engaging, but an engaging system is typically usable (O'Brien, Toms, 2008). To guide the case study, we formulated a specific applied research question as follows: In what ways can a location based MR game with indoor navigation can add value to the passenger experience at Zurich main station?

\subsection{Related work}

In general, it appears that certain behavioral phenomena such as "competition, self-mastery, social connection, tangible rewards, curiosity, purpose, playfulness, recognition and structure" (Traver, Sargent, 2011, pp. 38-40) motivate and engage people and enable them to stay on task even in achieving difficult long-term goals. Software that contain elements linked to the above-listed behavioral phenomena lead to varying degrees of adherence in all ages (such as games, social media). Given the context our research, an obvious example to consider is the well-known location based game Pokémon GO (Niantic, Inc, 2016). According Tang (2017), Pokémon GO's success is explained firstly because it induces nostalgia (invoking people's memory of Pokémon in the past); and secondly, the immersive location based storytelling takes the users outside and walk through the real-world, which is often a rewarding experience in itself (Tang, 2017). Dubbelman (2016) posits that narratives can indeed be 'engineered' (a notion author calls 'narrative game mechanics') to contain engaging, emotion-invoking elements (Dubbelman, 2016). In Dubbelman's (2016) explorations, examples of engaging story events include "building tension through spatial conflict, evoking empathy through characterization and creating moral dilemmas through player choices" (Dubbelman, 2016, p.39). 
Understanding cognitive factors in narrative design is important in identifying the fundamental elements of creating engaging experiences. In assessing cognitive factors, it is essential to consider individual and group differences as well (Çöltekin et al., 2019, Lokka, Çöltekin, 2019, Lokka, Çöltekin, 2020, Lokka et al., 2018). Similarly, in a digitally told interactive XR story, the design of visualization and interaction are important (Çöltekin et al., 2016, Schnur et al., 2018). In XR, a key visualization challenge is that virtual elements introduced to the scene occlude the real world objects (Çöltekin et al., 2020). In a location based implementation that requires navigating within a crowded indoor space, it is of key importance to consider solutions such as the use of transparency and 'warning mechanisms' for the viewer (audio or visual annotations, perhaps both) to prevent them from taking risks (Roxas et al., 2018).

Another challenging aspect of designing location based MR games (or interactive stories) is the interaction design (Çöltekin et al., 2020). Interaction design is challenging in all of XR (virtual, augmented and mixed reality systems); however they might be even more challenging for smartphones and tablets where the users need their hands to hold the device as opposed to those that are head-mounted (Hansberger et al., 2017). Current research demonstrates that MR-based (serious) games can be attractive and engaging (Dirin, Laine, 2018); although the current technology is laden with a number of human-computer interaction challenges (Stephanidis et al., 2019). A specific case study such as ours that attempts consolidating the proposed solutions into an implementation that is constrained by realworld demands will bring new knowledge and inform similar studies. Thus, for Zurich's main train station, where the context is well-defined, we designed a Mixed Reality (AR) app we call $S B B$ Stories. In this app, users can experience various stories that took place in the past based on early photographs taken at the station. With historical and artistic insights, and support of an indoor navigation solution, the app guides the users through the station. On this journey, the users can solve quests that guide them to the next location (Figure 1), which should support spatial learning. In this paper, we explain our process in creating the sample story "Das verlorene Spielzeug" (The lost toy). As we explain the story, we clarify and justify our design and implementation decisions based on additional literature and user feedback to guide others who may attempt designing and implementing similar apps; we report the findings from user feedback, and we share our insights.

\section{METHOD AND PROCESS}

\subsection{Target User Group}

For both of our objectives (to create an engaging story and support spatial learning), user characteristics are important, depending on their age group, professional status, technology familiarity etc., people learn differently and they will likely find different paradigms engaging (e.g., (Çöltekin et al., 2017, Schnürer et al., 2015). To identify a target user group we utilized the SBB's internal study that characterizes commuters based on observations and interviews (SBB AG, 2017). We cross-checked the SBB study with e.g., age groups that are active users of games such as Pokémon GO, to ensure that (lack of) familiarity with technology was not a major confound. We identified the target group called "Adventurer" by the SBB. In theory, Adventurers can be 15-49 year-olds (yo). However, SBB's statistics indicate that majority of Adventurers are 2039 yo, which broadly aligns with the profile that would be highly familiar with smartphones and tablets. People that fall into this segment also tend to be interested in arts, culture, literature and politics (SBB AG, 2017), which suggests that they may be willing to experience an interactive story that has historical elements in it. Thus we decided to keep the age bracket to 2039 yo.

\subsection{Game Concept Elaboration}

In the concept elaboration phase, a range of possible game concepts and ideas were considered not to limit creativity. We kept two conditions constant in all considered game concepts: The space would be Zurich main station, and players would move within this space. We explored various ideas, such as a puzzle concept we called "Puzzle Madness: A logic puzzle game" in which players needed to solve smaller puzzles in different parts of the station to create pieces of a bigger puzzle; another we called "The Rise of Wegmann: An adventure game" in which the original architect of Zurich main station Gustav Albert Wegmann (1812-1858) comes back to 'save' the station from danger; and another we called "Cubical: A multi-player building blocks game" in which multiple players would complete a puzzle collaboratively in the train station (there was an offline component too). We selected five of these ideas, and presented them to potential users $(n=97$, all in the target user category) in an online study to gauge interest and preferences (a "desirability study"). Based on the results of this survey and further iterations including expert input, we merged some of the ideas to two game concepts (i.e., SBB Puzzles, and SBB Stories). Because we take a user-centered design approach, we tested our ideas several times (three more times after the initial survey with 97 participants). In an interim study with participants $(n=7)$, we observed that the two concepts were preferred equally often. Given these responses, we integrated some puzzles within the SBB Stories concept, developed it further.

SBB Stories. In SBB Stories (a collection of historical stories), we exploit the fact that Zurich's main station has a rich history, which is partially documented in photographs, maps/plans, and old manuscripts. With some creativity, interactive stories that take place in the past can lead to an inspiring experience, and (re)discover unexpected places, information or cultural artifacts in the station too. Because one of the goals is to augment the user's spatial knowledge of the train station, this idea lends itself well for a location based MR (different than pure navigation or pure gaming).

\subsection{Technology}

Due to its prevalence and flexibility, we used the cross-platform game engine Unity (Glover, 2018); and since SBB already had an Android framework with indoor navigation features implemented, we worked with a combination of Unity with ARCore (Lanham, 2018). For the creation of the screen design and prototype, we used Figma (Figma, 2020), and for the overall visual style of the game, we decided to follow SBB's style guides to keep it coherent with their brand (SBB AG, 2020), and where needed, we styled them based on ARCore guidelines (Google Inc., 2019).

\subsection{User-Centered Design and User Evaluations}

As mentioned earlier, the prototypes of the app was user-tested at multiple stages of the development. Besides the desirability study in the beginning $(\mathrm{n}=97)$ and the decision study between two concepts $(\mathrm{n}=7)$; we conducted two more user studies on 
the prototype. The first of these was based on an interactive design prototype $(n=5$, age 18-29). The prototype was used offlocation to determine usability and clarity issues in the technology, interaction and narrative. In 30min sessions, participants completed predefined tasks on functionality (e.g., placing a virtual object on to a virtual surface), and navigated through the prototype on their own with the option to request help. We noted the number of times they needed help, and any issues they reported verbally. The prototype was adapted based on the feedback obtained from participants in this session. After tweaking the prototype, we conducted a second user study to measure usability and engagement with a functional prototype on-location. In this phase, participants $(n=6$, age 15-49, 3 men, 3 women) used the app on their own. Participants were selected to be in the Adventurer category of the SBB, and have some technology experience (e.g., 5 out of 6 participants were familiar with Pokémon GO). Participants were asked to "think aloud", which allows getting instant feedback about their preferences. Besides preference, we measured user engagement and usability based on standardized test instruments: User Engagement Scale Short Form (UES-SF) (Wiebe et al., 2014), and System Usability Scale (SUS) (Brooke et al., 1996). We also asked additional questions on the user experience, such as if scanning (when they held the phone up and scanned the environment to obtain images to support spatial registration) was a major disturbance or not. Sessions were audio, video and screen-recorded.

\section{RESULTS}

The main result of this research is a validated XR game concept and a functional prototype of a location based MR app that allows its users 'travel' back in time. The following sections detail various parts of the game concept and the prototype. Based on theoretical and practical considerations and user feedback, we designed the SBB Stories as such that the player can experience interactive stories with an emphasis on historical events, and visit unforeseen places on an adventure through the station. While playing the game, a user needs solve 'quests' throughout the story which should trigger a sense of self-mastery and competition (Figure 1). As an additional incentive, there are tangible rewards: A user can collect points by finishing quests, and when they collect enough points, they can trade them for a variety of vouchers usable at Zurich main station.

$S B B$ Stories uses an experience-based theme (as opposed to truth-based theme, see (Schell, 2015)) that focuses on two main parts: Adventures for engaging the users, and the fusion of past and present for an inspirational experience. The game takes the visitors out of their daily routine and take them on an adventure through the railway station. Many different stories can be followed, depending on interest, time and location. When embarked on an adventure, some quests and riddles are presented along the way. Some stories are fictional, while others are documentaries. As a deliberate motivational component, we tweaked the design to make the users should feel as curious explorers, seeking the unknown parts of the railway station. Inspired by Alexandre Devaux" "Time travel!" implementation (Devaux, 2018) to a degree, besides our own brainstorming and user feedback that indicated arts and culture may be interesting for our target audience, we decided to fuse historical images into the real world (Figure 2). By merging past and present together, the gamified interactive story offers historical insights and potentially give the users nostalgic feelings. This
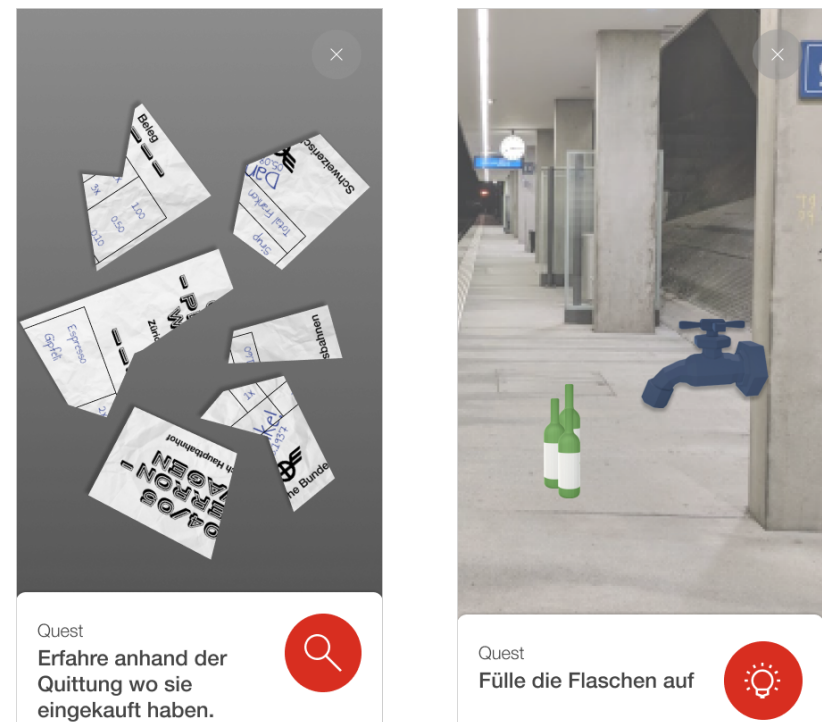

Figure 1. Sample quests. Left: 2D/AR quest "Find out where the receipt was from", Right: AR/MR quest "Fill the bottle".

decision was motivated by the well-understood notion that triggering emotion is a key feature in engagement, adherence and memorability (Nilsen et al., 2004).

Goal types. We designed two goal types for the set of interactive stories in SBB Stories: Player goals (designed based on theories of engagement and user feedback), and Learning goals (driven by our goal to improve the user experience in the station and improve spatial knowledge acquisition). Player goals include solving all the quests and stories to complete the game, and collect all the achievements in the game; whereas the Learning goals are about transmitting knowledge about historical and cultural insights as well as about services and offers found in Zurich main station.

Interaction. Interaction design is one of the biggest challenges in user acceptance and adherence to XR experiences, and it is critical to their success as a new medium (Stephanidis et al., 2019). Our game concept includes two types of interactions: direct and indirect. Direct interactions represent actions from the real world, e.g., picking up a water bottle and filling it from a virtual tap. The phone mimics behaviour of a real water bottle by using the camera and spatially placing a virtual 3D water bottle. Indirect interactions define non-realistic actions such as the press of a button. These were designed based on the state-of-the art interaction functions available in smartphones and tablets and with the goal to keep them as simple as possible (e.g., single click) and users were guided regarding the interactions available to them. We also paid attention that the users do not suffer from the so-called "gorilla arm" effect (Hansberger et al., 2017), that is, they would not have to hold the phone/tablet away from their body for extended amounts of time, which is ergonomically sub-optimal (the shoulders/arms/neck hurts).

Aesthetics. As mentioned earlier, the aesthetics choices in the game follow design guidelines from the SBB, as the research is conducted collaboratively with the SBB. Furthermore, the design guidelines for AR/MR applications (Google Inc., 2019) offered many insights for creating a positive user experience, such as using markers that point towards invisible AR/MR elements, which is critically important to control the complexity of hidden interactions and navigate the MR scene. 

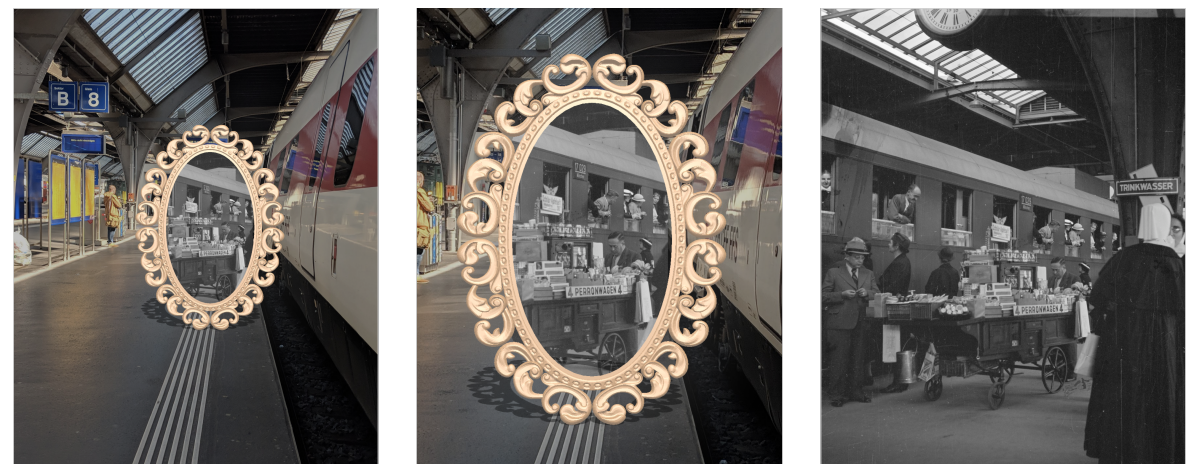

Figure 2. Portal perspectives. Left: Portal at a distance, Middle: Near the portal, Right: Inside the portal.

Components and modular design. The concept of the game consists of modular components that can work independently from each other. This structure allows to exchange and adapt the concept and allows to add components easily. The following paragraphs describe several important components and their usage in the game.

Map: Because the game involves moving around in the station, we implemented a map showing Zurich main station, as a base component in the game. On the map, different interactive entry points (stories, see subsection Story) in different locations are marked (Figure 3). Users can see their own position and navigate to a certain entry point. The navigation can be either in $2 \mathrm{D}$ or in AR/MR mode. As soon as a user gets close to an entry point, they can switch to the AR/MR mode to start a story.

Portal: A very important component that marks where the story episodes can be accessed is the 'the portal'. The portal component is placed in AR/MR and contains a historical image within the portal (Figure 2). When the users move close to a portal, they can enter it and view the picture entirely. We have chosen the portal as a key game component to provide the user with a window to the past. Similar to a gallery in real world, users can watch the images from outside but also have the possibility to 'go in' and travel back in time.

Visualization. Since we use a video see-through MR in the interactive story, visualizations of virtual objects blend with the real world. To avoid accidents and other disturbance in a potentially crowded space such as the main train station of the largest city in Switzerland, we decided to design the MR interactions with virtual objects when the user was stationary. When an episode of the story was completed, the user moved to another location using the Map feature and triggered the next episode using the Portal feature. Besides the concerns about occlusion, we have paid close attention in the integration of the historical views to the current space and framed the portals to mark and embed the visual transitions.

Narrator: As a familiar story-telling component, there are different text elements in the game in selected points in time and positions that the user can either read or listen to. Those texts are usually visible in combination with an image. An audio version of the narrator enables the users to focus on the images while listening to the story.

Achievements and Rewards: Because tangible rewards, selfmastery and competitive elements in the game are important, we designed the interface to include feedback on achievements and rewards. The selected target group (see Target Group 2.1) shows a preference for rewards. Therefore, within the game, users can get achievements while playing stories and eventually trade them against certain rewards. Rewards may be coupon codes for the railway system, for an offering at the Zurich main station or similar.

Story. A Story in SBB Stories app is an encapsulated playable adventure that leads the user through different parts of the train station while completing quests. While some stories are fictional, others might tell a real story that happened in the past in that location. The following sample story is a fictional story, created to showcase the application.

Sample Story: The sample story "Das verlorene Spielzeug" (The lost toy) is about a child who has lost her toy while walking through the railway station. She and her family are about to board the Orient Express to Athens, which departs shortly. It is the player's quest to find the toy and return it to the girl before the train departs.

Timeline: The story consists of five parts; each of them contains a location and a variety of actions. When the users finish all the components of one location, they are requested to move to the next one. This timeline was built in this composition to follow the interest curve: This curve represents a 6-step progress of how interest should be handled over time to remain attractive to the viewer (Schell, 2015).

Quests: In the general game concept, there are two types of quests: 1) 2D quests where the user's location has no affect on the game, i.e., more AR than MR, or one can even solve them on the device display, and the quest is solvable in a relaxing hand position to prevent the "gorilla arm" effect 2) MR quests which require the player to move around in space, i.e., they are location-dependent. Those two game types were defined to provide variety to the users, provide some control on possible ergonomics problems, and prevent mishaps due to visual occlusion. In the sample story, two quests are available: Receipt Puzzle and Fill the bottle:

- Receipt puzzle (2D/AR quest): The players have to piece back together a receipt in order to find out more information about the story (Figure 1, left).

- Fill the bottle (3D/MR quest): The players have to grab a water bottle and fill it up with water (Figure 1, right).

Historic content and artistic insight: The story plays in the year 1937 and contains multiple references (e.g., hand written receipts, prices, old logos) that were common at this time. The 


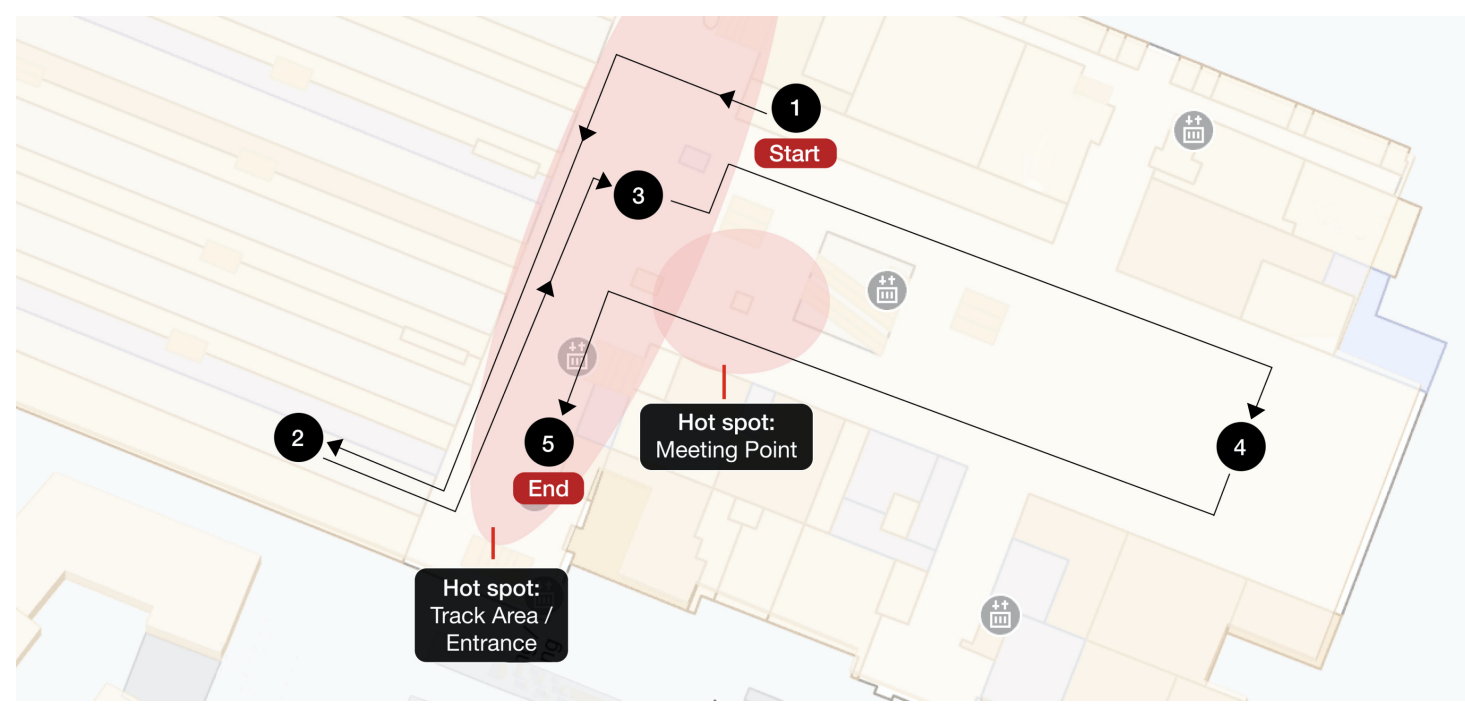

Figure 3. Sample story locations (black dots) and potential hot spots (pink overlay). Ground floor, Zurich main station.

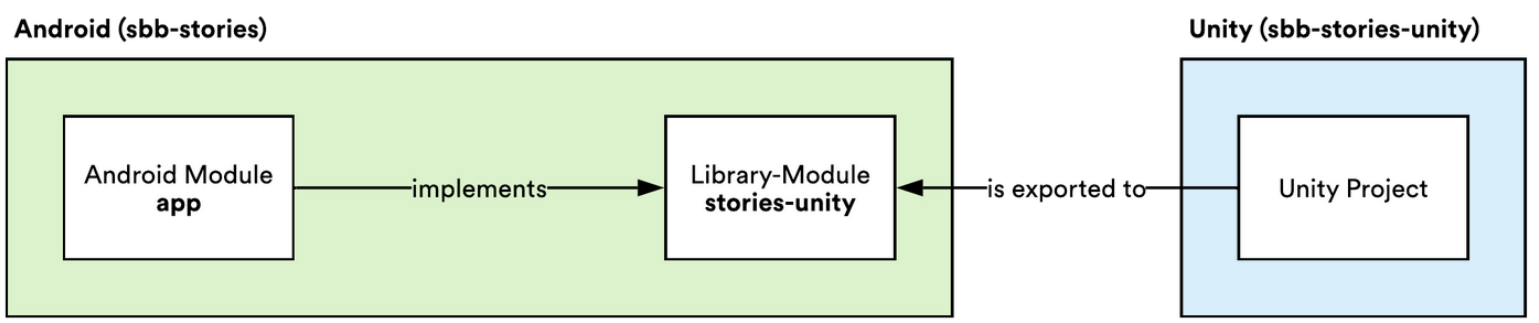

Figure 4. Implementation architecture.

player gets to know that the family is departing with the Orient Express from Zurich to Athens, which was a valid route during this period (Smith, 2019). Furthermore, the prices that are visible on the receipt (partly visible in Figure 1) were adapted to match the year 1937 (50 cents per pound) (Martin, 1954). Furthermore, the timeline revealed that the story contains information about an art piece: The Century Ball, a golden ball, created by Dieter Meier and located in the main hall in Zurich main station (SBB, 2020).

Location: The story takes place on the ground level of the Zurich main station. The main hall is a large open space, its capacity and centrality makes it an attractive venue for events; however, besides these events, it is not extremely crowded. Figure 3 displays a section of the ground floor of Zurich main station with the five story locations. The lines describe suggested walking paths which were designed based on literature research and our observations. It has been previously shown that in such spaces, people often stay longer around information boards (Nakamura et al., 2005).

Furthermore, the platforms, especially at the top end, tend to get very busy at peak times (Schomborg, Waßmuth, 2013). Therefore such hot spots with expected above-average traffic (indicated in pink overlays in Figure 3) should be avoided. However, for the sample story, those spots were chosen on purpose to convey the hectic emotion represented within the story. The user asks strangers for help in Location 3, and sees the family depart among many other passengers from the station in Location 5 (Figure 3).
Prototype. The resulting implementation of the prototype consists of two modules, an Android project (sbb-stories) and a Unity project (sbb-stories-unity). Both projects were individually developed. By exporting the Unity project into an Android library, we could synchronize the two projects (Figure 4). As shown in Figure 4, we have designed the software architecture so that the Unity project contains the AR content, and manages the flow of the story. As a small but important technical contribution on its own, our software architecture handles the communication between the two modules, and therefore sends and receives messages from the Android module. Whenever there are changes in the Unity project, the Unity code will be exported into a library-module called stories-unity that is included and used within the Android project.

The Android project contains two modules: The Android module app and a library stories-unity. The app module contains the implementation of the user interface (UI) components such as the Map or Achievements and Rewards. The Android module also contains all of the textual and audio story content that will be shown or played whenever requested by the Unity content. The stories-unity module contains the exported Unity project as well as some added interface classes for handling the communication. This separation allowed to use Unity for rendering the AR content and Android for flat UI elements. Therefore, the core functionality of both systems could be used in an efficient way. Furthermore, the application architecture was chosen with modularity and scalability in mind. The implemented features, such as the Map, Achievements and Stories, are independent components that do not rely on each other. Another component worth highlighting here is the Pipeline component, which was 
created for building stories easily. This component (implemented as a ScriptableObject in Unity), allows adding the needed elements for a story with drag and drop within Unity. This concept allowed to extract a shortened version of the sample story for a demonstration in a matter of minutes.

Usability and Engagement. Besides the cycles of user tests throughout the project that have been described earlier, we conducted final usability and engagement tests $(n=6)$ for the prototype implementation (see section 2.4) where we collected quantitative responses from the participants based on SUS and UES-SF, as well as qualitative insights on how they used and interacted with the final prototype in-situ (on-location). Overall quantitative results are encouraging (Figure 5).

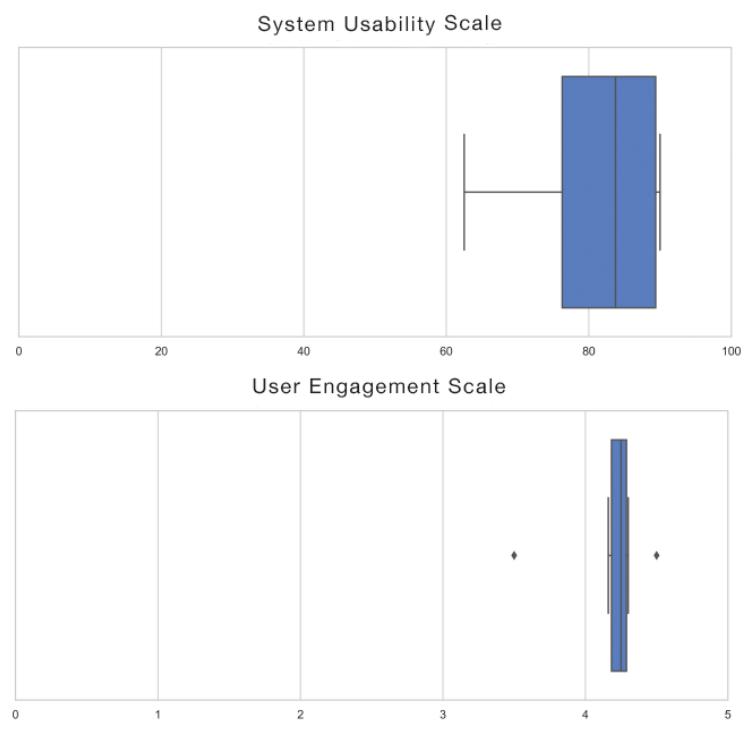

Figure 5. Participants ranked the prototype as usable and engaging. Top: Outcome of the SUS (in percent). Bottom: Outcome of the UES-SF (Likert scale 1-5).

The SUS score was $83.75 / 100$, and the UES score was 4.25/5.00, which are both high scores (O'Brien, Toms, 2008, Bangor et al., 2009). Also, all participants confirmed that they would spend extra time at the station to play the game, which supports this quantitative finding. Even though we have a small sample, these are standardized tests that are designed to control for some of the possible participant bias, and we believe they point in the right direction also because the qualitative observations overall support these numbers. However, qualitative observations also suggest some points of improvements. A key issue we detected was about MR interactions: When entering a portal, all participants realised how they can read the text and how to continue with the game. However, only 2 out of 6 participants entered the portal at expected time points, while others stayed "outside" at least once during the game. Another important issue we observed was about the navigation $v s$. visualization: Participants walked rather fast towards objects in MR, and therefore sometimes missed the portal entry. An additional related problem was that some participants misjudged their distance to the virtual objects. However, the markers helped the participants to find the virtual objects if they were not visible on the screen. Last but not least, somewhat surprisingly, 5 out of 6 participants stated that they preferred an experience without audio narrations, and they reported that 'scanning' (holding the device to scan the space for spatial referencing in MR) was a positively interesting experience.

\section{DISCUSSION}

We conceptualized, developed, implemented and user-tested an fictitious interactive XR story that takes place in the past based on actual historical photographs of the Zurich main station. The entire project was based on a user-centered design approach. Our initial study with 97 participants demonstrated that there was enthusiasm for an MR game that has elements of arts and culture, and is location based. Our interim studies informed the prototype development and helped narrow our focus, along with the relevant literature. The usability and engagement studies suggest that our XR game concept overall both usable and engaging. In this section, we interpret our findings in the light of relevant literature.

The ratings for SUS (83.75/100) may be fairly robust as for most usability studies, six, even five participants are shown to detect usability issues (Nielsen, 2012). A clear usability issue that was detected in this study was about the Portal feature. The fact 4 out of 6 participants omitted 'clicking' these portal images at least once in the game, and seemingly not realizing that these images were spatially referenced to their current position presents a serious (though solvable) usability issue. Most likely explanation for this is that the four participants who did not go through the portal at the expected time point were not able to interpret the way the portal was visualized: The portals were visualized as antique picture frames, and it seems like without further annotation, it was not obvious to these participants that they could "step inside" these portals (see screen 1 in Figure 2 ). This issue could be solved by adding annotations, explicit instructions, or a brief training session at the beginning of the game. For example, this issue did not occur in the interim testing, because in that case participants received explanations in the beginning (in the final test, we were keen to measure if participants could use the app without help). Secondly, as one participant remarked, the portals are rather small. A larger, more prominent portal entrance may also help. Thirdly, the users may have been overwhelmed by the experience or distracted by the various multimedia elements: Once the user got near a portal, narration started to play, and text appeared. The participants that stayed outside of the portal possibly focused on those newly appeared components and forgot about the portal images. The fact that 5 out of 6 participants preferred an experience without audio narrations is also interesting. It appears that in our implementation, reading was faster than listening the audio narrations until the end. We believe this can be solved by making this feature optional: If the users have the opportunity to both listen and read, they may choose what is more efficient or pleasant for them in the moment. Another usability issue was about judging distances. It appears that people misjudged their distance to the virtual objects in our study. This phenomenon has been shown previously in other studies (Çöltekin et al., 2016) and is a known problem with spatial interaction in general. We observed that participants walked with a high pace towards the XR objects, possibly because they want to be efficient in the game. Even though they reported that they were familiar with Pokémon GO, they were still facing problems with XR elements while playing the game. A possible solution for this problem might be to adapt the interaction, so that it allows the user to move with more freedom, while still be able to continue in the story. A more interactive on-boarding (training) where users get comfortable with spatial interaction might help as well.

Arguably, some of the above-mentioned issues may be removed by using a head-mounted XR display instead. However, the 
smartphone was "immersive enough", i.e., interestingly, it was not always obvious to the the participants whether the object they were looking for was in XR or in the real world. For example, some participants were looking for the Jahrhundertkugel in XR, even though it is a physical object. More precise wording and guidance for the user, or using artificial/abstract visualizations, or more transparency could reduce this misunderstanding. Some of the known 'tricks' in interaction design, specifically, the addition of the marker (see section Aesthetics) guided participants well when the XR object was not in sight. In the earlier user tests, participants were asked to place virtual objects on planes that were created by the XR framework, which was confusing for them. The implementation of automatic placement made this interaction more clear and less confusing for the participants. Conversely, the automatic placement allowed to put the objects outside of a certain boundary around the user. This boundary solved the issue of having users place the objects below themselves. We measured not only usability, but also engagement in the final study. The engagement score is high (4.25/5.00), but unlike usability testing where approximately 6 users are deemed enough, we do not have a reassuring "sample size" for UES-SF. In our case, besides the high quantitative score, all 6 participants told us that they would use more time at the station just to play this game, thus we believe the engagement levels were indeed high.

A future study that uses UES-SF with more participants, record eye movements (e.g., (Çöltekin et al., 2014)) and possibly obtain physiological measures to understand levels of alertness, anxiety and/or positive emotional experience would make these observations more robust. A general concern with the current concept of SBB Stories, as mentioned earlier, is safety. Even though we limited the XR interactions on specific locations, when people walked between these locations, they were walking fast. This can be a potential safety hazard in a crowded space such as the main station of the city. Before the game reaches a broader community, safety improvements must be carefully considered. Based on careful design of the indoor navigation instructions and an in-depth analysis of the passenger flows of the main station, the XR objects should be placed in safe, not-crowded locations. Moreover, the app should notice whenever a user is walking and eventually limit the features in this state to prevent incidents. On a technical note, image recognition did not work unless the reference images were replaced every day (due to dirt and varying lighting conditions). Interestingly participants regarded the scanning experience a positive one despite having to do it multiple times in some cases, possibly due to its novelty. Note that this technology (scanning) works best for diversified textures; therefore, it might be more suitable to use it for story elements that have more diverse texture, and prefer other forms of spatial referencing in work similar to ours.

\section{CONCLUSION AND OUTLOOK}

The game concept we featured as a case study SBB Stories reached the goals defined by the research team and by the users and 'added value' in terms of increased spatial awareness, usability and user engagement. A general lesson we have learned was that when combining historical content with the present, it is important to identify appropriate transition cues between the two, and consider design solutions. Our portal interaction led to somewhat mixed results in terms of intuitiveness but the SBB Stories overall provided a favorable proof of concept. Consequently, upon some refining, attractive and engaging histor- ical exploration tools that also enhance spatial knowledge acquisition for indoor spaces similar to Zurich's main train station is possible. Another take away from this effort is that when designing an XR game, choosing which game elements should be $\mathrm{XR}$ and which content could be presented in $2 \mathrm{D}$ requires care. While it may be exciting to use XR for every game element, it is worth considering the limitations of the users (e.g.,, arm strength, technical skills, age). It is also worth noting a methodological observation: For testing XR applications, the think aloud approach was very useful as participants' hands are busy. Further possible improvement of the prototype that would require additional research on waiting times (Çöltekin, Reichenbacher, 2011), and the indoor navigation component that we did not discuss in the scope of this paper. By using indoor navigation to place $\mathrm{XR}$ objects more precisely, the fusion of historical images and the present scene can be improved. The effects of additional interaction and visualization concepts (e.g., a larger portal visualization, making audio vs. text narration optional, adding 'collectibles' to make people return to the game, etc.), and providing some training opportunities should be further tested as well. Last but not least, we see signs of the increased spatial awareness in our tests, which we interpret as new spatial knowledge acquisition, which should also lead to better navigation. These promising first observations can be tested more systematically in terms of how much they affect new spatial knowledge acquisition, e.g., over time, or for user groups with different characteristics. After resolving the feature limitations, further episodes to the same story, and further stories should be added to the game. The concept of this game takes multiple story types into account, such as seasonally themed or branded stories. By making use of different story types, more locations in the station (more services and offers) could be discovered by the users and they could learn the space around them better, navigate more efficiently, and would be potentially even more engaged in the app to discover more.

\section{ACKNOWLEDGEMENTS}

We are grateful to the SBB's Extended Reality team for partially funding this project.

\section{REFERENCES}

Bangor, A., Kortum, P., Miller, J., 2009. Determining what individual SUS scores mean: Adding an adjective rating scale. Journal of Usability Studies, 4(3), 114-123.

Brooke, J. et al., 1996. SUS-A quick and dirty usability scale. Usability Evaluation in Industry, 189(194), 4-7.

Çöltekin, A., Demsar, U., Brychtová, A., Vandrol, J., 2014. Eye-hand coordination during visual search on geographic displays. CEUR Workshop Proceedings, CEUR-WS, 12-16.

Çöltekin, A., Reichenbacher, T., 2011. High quality geographic services and bandwidth limitations. Future Internet, 3(4), 379396.

Çöltekin, A., Brychtová, A., Griffin, A. L., Robinson, A. C., Imhof, M., Pettit, C., 2017. Perceptual complexity of soillandscape maps: a user evaluation of color organization in legend designs using eye tracking. Int. Journal of Digital Earth, 10(6), 560-581. 
Çöltekin, A., Griffin, A. L., Slingsby, A., Robinson, A. C., Christophe, S., Rautenbach, V., Chen, M., Pettit, C., Klippel, A., 2020. Geospatial information visualization and extended reality displays. Manual of Digital Earth, Springer, 229-277.

Çöltekin, A., Lokka, I.-E., Zahner, M., 2016. On the usability and usefulness of 3D (geo) visualizations - A focus on virtual reality environments. Int. Archives of the Photogrammetry, Remote Sensing Spatial Information Sciences, XLI-B2, 387-392.

Çöltekin, A., Oprean, D., Wallgrün, J. O., Klippel, A., 2019. Where are we now? Re-visiting the Digital Earth through human-centered virtual and augmented reality geovisualization environments (Editorial). Int. Journal of Digital Earth, 12(2).

Devaux, A., 2018. Time travel! https://twitter.com/Alexandre Devaux/status/1070333933575917569 (last accessed 5/2/ 2020).

Dirin, A., Laine, T. H., 2018. User experience in mobile augmented reality: Emotions, challenges, opportunities and best practices. Computers, 7(2), 33.

Dubbelman, T., 2016. Narrative Game Mechanics. Int. Conference on Interactive Digital Storytelling, 39-50.

Figma, 2020. Figma: The collaborative interface design tool. https://www.figma.com (last accessed 5/2/2020).

Glover, J., 2018. Unity 2018 Augmented Reality Projects: Build four immersive and fun AR applications using ARKit, ARCore, and Vuforia. Packt Publishing Ltd.

Google Inc., 2019. Augmented Reality Design Guidelines. https://designguidelines.withgoogle.com/ar-design/augmentedreality-design-guidelines/introduction.html (last accessed $5 / 2 / 2020)$.

Hansberger, J. T., Peng, C., Mathis, S. L., Shanthakumar, V. A., Meacham, S. C., Cao, L., Blakely, V. R., 2017. Dispelling the gorilla arm syndrome: The viability of prolonged gesture interactions. Int. Conference on Virtual, Augmented and Mixed Reality, Springer, 505-520.

Lanham, M., 2018. Fundamentals of Google ARCore: Learn to build augmented reality apps for Android, Unity, and the web with Google ARCore 1.0. Packt Publishing Ltd.

Leimgruber, P., SBB, 2017. Smart station. https://challenge. digitalswitzerland.com/portfolio/smart-station (last accessed $5 / 2 / 2020$ ).

Lokka, I. E., Çöltekin, A., 2019. Toward optimizing the design of virtual environments for route learning: Empirically assessing the effects of changing levels of realism on memory. Int. Journal of Digital Earth, 12(2), 137-155.

Lokka, I. E., Çöltekin, A., 2020. Perspective switch and spatial knowledge acquisition: effects of age, mental rotation ability and visuospatial memory capacity on route learning in virtual environments with different levels of realism. Cartography and Geographic Information Science, 47(1), 14-27.

Lokka, I. E., Çöltekin, A., Wiener, J., Fabrikant, S., Röcke, C., 2018. Virtual environments as memory training devices in navigational tasks for older adults. Sci. Reports, 8(1), 1-15.

Martin, F. H., 1954. A History of Coffee Prices in the United States, 1840-1954. Monthly Labor Review, 77(7), 765-767.
Nakamura, K., Zhao, H., Sakamoto, K., Suzukawa, N., 2005. Visualizing passenger flow in railway station using laser scanners. Proceedings of the 9th Computers in Urban Planning and Urban Management Conference (CUPUM).

Niantic, Inc, 2016. Get up and go: Catch Pokémon in the Real World with Pokémon GO! https://www.pokemongo.com (last accessed 5/2/2020).

Nielsen, J., 2012. How many test users in a usability study. Nielsen Norman Group, 4(06).

Nilsen, T., Linton, S., Looser, J., 2004. Motivations for augmented reality gaming. Proceedings of FUSE, NZGDC'04, June 26-29, 2004, Dunedin, New Zealand, 4, 86-93.

O’Brien, H. L., Toms, E. G., 2008. What is user engagement? A conceptual framework for defining user engagement with technology. Journal of the American Society for Information Science and Technology, 59(6), 938-955.

Roxas, M., Hori, T., Fukiage, T., Okamoto, Y., Oishi, T., 2018. Occlusion handling using semantic segmentation and visibilitybased rendering for mixed reality. Proceedings of the 24th ACM Symposium on Virtual Reality Software and Technology, 1-8.

SBB, 2020. The century ball. https://www.sbb.ch/en/stationservices/at-the-station/railway-stations/shopville-zuerich-main -station/artwork/boule-dor-centenaire.html (last accessed 5/2/ 2020).

SBB AG, 2017. Besser ankommen. SBB AG.

SBB AG, 2020. Alles zu den digitalen Anwendungen der SBB. https://digital.sbb.ch (last accessed 18/1/2020).

Schell, J., 2015. The Art of Game Design: A Book of Lenses, 2nd. Ed. CRC Press.

Schnur, S., Bektaş, K., Çöltekin, A., 2018. Measured and perceived visual complexity: A comparative study among three online map providers. Cartography and Geographic Information Science, 45(3), 238-254.

Schnürer, R., Sieber, R., Çöltekin, A., 2015. The next generation of atlas user interfaces: A user study with digital natives. Modern Trends in Cartography, Springer, 23-36.

Schomborg, A., Waßmuth, V., 2013. Stuttgart S21 Analyse der Fußgängerströme. https://tinyurl.com/ya7k6ahq (last accessed $5 / 2 / 2020$ ).

Smith, M., 2019. A history of the Orient Express. https://www. seat61.com/OrientExpress.htm (last accessed 5/2/2020).

Stephanidis, C., Salvendy, G., Antona, M., Chen, J. Y., Dong, J., Duffy, V. G., Fang, X., Fidopiastis, C., Fragomeni, G., Fu, L. P. et al., 2019. Seven HCI grand challenges. Int. Journal of Human-Computer Interaction, 35(14), 1229-1269.

Tang, A. K. Y., 2017. Key factors in the triumph of Pokémon GO. Business Horizons, 60(5), 725-728.

Traver, K., Sargent, B. K., 2011. The Healthiest You: Take Charge of Your Brain to Take Charge of Your Life. Simon and Schuster.

Wiebe, E. N., Lamb, A., Hardy, M., Sharek, D., 2014. Measuring engagement in video game-based environments: Investigation of the User Engagement Scale. Computers in Human Behavior, 32, 123-132. 